Chirurg 2016 $87: 889$

DOI 10.1007/s00104-016-0281-3

Online publiziert: 1. September 2016

๑) Springer-Verlag Berlin Heidelberg 2016

CrossMark

M. Schrempf $\cdot$ M. Anthuber

Klinik für Allgemein-, Viszeral- Transplantationschirurgie, Klinikum Augsburg, Augsburg, Deutschland

\title{
Vollremission durch Radiochemotherapie beim Rektumkarzinom - Abwarten oder Operieren?
}

lich ist. Für die Auswertung wurde der Signifikanzwert $p=0,01$ gewählt.

Renehan AG, Malcomson L, Emsley R et al (2016) Watch-and-wait approach versus surgical resection after chemoradiotherapy for patients with rectal cancer (the OnCoRe project): a propensity-score matched cohort analysis. Lancet Oncol 17:174-183

Hintergrund. Die neoadjuvante Radiochemotherapie (nRCT) senkt beim tiefsitzenden, lokal fortgeschrittenen Rektumkarzinom die Rate an Lokalrezidiven und erhöht die Wahrscheinlichkeit des Sphinktererhalts. Berichte über Patienten mit einem vollständigen Ansprechen („complete response“, CR) nach nRCT gaben Mitte der 2000er Jahre den Anstoß zu Diskussionen über die onkologische Sicherheit der Watch-and-Wait-Strategie nach CR im Vergleich zur Resektion.

Methoden. Für die „Oncological Outcomes after Clinical Complete Response in Patients with Rectal Cancer (OnCoRe)-Studie“ wurden Patienten mit einem Adenokarzinom des Rektums und nRCT eingeschlossen. Um den Einfluss möglicher Störfaktoren zwischen den beiden Gruppen „Watch-and-Wait“ und „Resektion“ zu minimieren wurde ein „propensity score matching“ angewendet. Primärer Endpunkt war das krankheitsfreie Überleben. In der Arbeit wurden pelvine Lokalrezidive („pelvic recurrence") und erneutes lokales (endoluminales) Tumorwachstum („local regrowth“) unterschieden, wobei letzteres nicht für den primären Endpunkt berücksichtigt wurde, da dieses einer Salvage-Therapie zugäng-
Ergebnisse. Von 259 Patienten hatten 31 ein CR nach nRCT. Weitere 98 Patienten mit CR wurden über Registerdaten in die Watch-and-Wait-Gruppe eingeschlossen. Von diesen 129 Patienten entwickelten 48 (37\%) nach einer medianen Nachbeobachtungszeit von 33 Monaten ein Rezidiv (endoluminales Rezidiv $n=$ 41; endoluminales Rezidiv und Fernmetastasen $n=3$; nur Fernmetastasen $n=$ 4).

Zwischen der Watch-and-Wait- und der Resektionsgruppe (109 Patienten je Behandlungsgruppe) zeigte sich ohneBerücksichtigung des „local regrowth“ kein Unterschied im krankheitsfreien 3-Jahres-Überleben $(88 \%$ in der Watch-andWait-Gruppe vs. $78 \%$ in der Resektionsgruppe $p=0,043)$. Auch im 3-JahresGesamtüberleben war kein signifikanter Unterschied nachweisbar $(96 \%$ in der Watch-and-Wait-Gruppe vs. $87 \%$ in der Resektionsgruppe $p=0,024)$. Das kolostomafreie 3-Jahres-Überleben war in der Watch-and-Wait-Gruppe signifikant geringer $(p<0,0001)$.

Diskussion und Fazit. Eine Watch-andWait-Strategie kann bei CR nach nRCT für ausgewählte Patienten eine sinnvolle Alternative zur Resektion darstellen. Allerdings ist ein CR nach nRCT mit ca. 10-12\% aller Fälle sehr selten. Daher sollte beim Patienten nicht die Hoffnung geweckt werden, dass durch alleinige RCT zuverlässig eine Operation vermieden werden kann. Die Befürworter der
Watch-and-Wait-Strategie empfehlen in den ersten Jahren ein engmaschiges Follow-up bestehend aus klinischer Untersuchung, CEA-Bestimmung, Endoskopie, MRT und CT. Dieses Vorgehen ist nicht nur mit hohen Kosten verbunden, sondern setzt auch eine entsprechende Patientencompliance voraus. Es sollte zudem die Möglichkeit in Betracht gezogen werden, dass das Ansprechen auf nRCT selbst ein prognostischer Marker sein könnte und idealerweise im Studiendesign berücksichtigt werden [1]. Daten zum Outcome nach SalvageTherapie sind spärlich und erfordern längere Nachbeobachtungszeiten. Daher sind weitere prospektive Studien nötig, um eindeutige Empfehlungen zur Watchand-Wait-Strategie geben zu können.

\section{Korrespondenzadresse}

\section{Prof. Dr. M. Anthuber}

Klinik für Allgemein-, Viszeral- Transplantationschirurgie, Klinikum Augsburg

Stenglinstr. 2, 86156 Augsburg, Deutschland matthias.anthuber@klinikum-augsburg.de

Interessenkonflikt. M. Schrempf und M. Anthuber geben an, dass kein Interessenkonflikt besteht.

\section{Literatur}

\footnotetext{
1. Maas M, Nelemans PJ, Valentini Vet al (2010) Longterm outcome in patients with a pathological complete response after chemoradiation for rectal cancer: a pooled analysis of individual patient data. Lancet Oncol 11:835-844
} 